\title{
DESIGN, SYNTHESIS AND COX1/2 INHIBITORY ACTIVITY OF NEW QUINAZOLINE-5-ONE DERIVATIVES
}

\author{
Mohammed A. Hara ${ }^{a}$, Mostafa H. Abdelrahman ${ }^{a}$, Ahmed S. Aboraia ${ }^{b, \star}$, Mohamed M. Amin ${ }^{c}$, \\ Osama I. El-Sabbaghab ${ }^{d, e}$ \\ ${ }^{a}$ Department of Organic Chemistry, Faculty of pharmacy, Al-azhar University, Assuit Branch, 71524, Assuit, \\ Egypt. mohammedhara79@yahoo.com, mhamed102004@yahoo.com \\ ${ }^{b}$ Department of Medicinal Chemistry, Faculty of Pharmacy, Assuit University, 71524, Assuit, Egypt. \\ Ahmed.mohamed15@pharm.au.edu.eg \\ 'Department of Organic Chemistry, Faculty of Pharmacy, Suez Canal University, 41522, Ismailia, Egypt. \\ msaid123eg@yahoo.com, mohamed_said1@pharm.suez.edu.eg \\ ${ }^{d}$ Departmentof Medicinal Chemistry, Faculty of Pharmacy, Zagazig University, Zagazig, Egypt. \\ osamaelsabbagh@yahoo.com \\ ${ }^{e}$ Department of Pharmaceutical Chemistry, Faculty of Pharmacy, Taif University, 888 Al-Haweiah, Taif 21974, \\ Saudi Arabia. o.elsabbagh@tu.edu.sa \\ "Author to whom correspondence should be addressed
}

\section{ABSTRACT}

A new series of 1-(4-Acetylphenyl)-7,7-dimethyl-3-(substitutedphenyl)-1,2,3,4,7,8-octahydroquinazolin-5(6H)-ones (6-15) were synthesized and tested against COX-1 and COX-2 enzymes. Those compounds exhibited strong interaction at the COX-2 binding site and poor interaction at the COX-1 active site. Subjected to in vitro cyclooxygenase COX-1/COX-2 inhibition assay; most of the compounds especially compounds $6,7,12,13$, and 16 exhibited potent anti-inflammatory effects, selective COX-2 inhibition, with half-maximal inhibitor concentration (IC50) values of $0.22-1.42 \mu \mathrm{M}$ and selectivity index (SI) values of 6.16-14.18 compared with celecoxib (IC50 $=0.05 \mu \mathrm{M}$ and COX-2 SI: 296), diclofenac (IC50 = $0.8 \mu \mathrm{M}$ and COX-2 SI: 4.87), and indomethacin (IC50 $=0.49 \mu \mathrm{M}$ and COX-2 SI: 0.08) as reference drugs. Docking study has been carried out to confirm the binding affinity and selectivity of the most active compound (compound 6) to COX-2 enzyme.

\section{Indexing terms/Keywords}

COX-2 selective; Quinazolinone; COX-1/2 Inhibitory; Docking.

\section{INTRODUCTION:}

Pain and inflammation are very commonly associated with numerous pathological conditions commonly prescribed with nonsteroidal anti-inflammatory drugs (NSAIDs). Most of the NSAIDs available in the market are known to inhibit isoforms of the enzyme cyclooxygenase (COX), a constitutive form COX-1 which is responsible for main side effects of antiinflammatory such as edema, abdominal colic and peptic ulcer, and an inducible (pathological) form COX-2 which responsible for therapeutic effects. There is continuous development of new drugs that have potent anti-inflammatory activity, with minimum side effects and high safety margin $(1,2)$. The design of effective agents that have fast action and provide relief from pain and inflammation is a major challenge for medicinal chemists such as our novel quinazolinone as non-acidic NSAIDs.

Cyclic $\beta$-diketones are an important class of organic compounds, one of which is 5,5-dimethyl 1,3-cyclohexandione (Dimedone) which is used as a precursor for the preparation of different intermediates such as enaminones and different heterocyclic rings such as quinazoline, quinoline, acridine and hydrazone derivatives that have interesting biological activities. The choice of quinazolinone heterocycle as a central core was fundamental due to its presence in the potent anti-inflammatory natural alkaloids rutaecarpine1 and tryptanthrin2 (3).

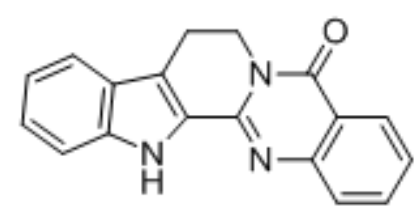

Rutaecarpine (1)<smiles>O=C1c2ccccc2-n2c1nc1ccccc1c2=O</smiles>

Tryptanthrin (2)

The presence of quinazoline moiety in the aforementioned natural alkaloids, which can undergo substitution at the heteroatom or the aromatic ring, is a necessary requirement for the anti-inflammatory activities $(4,5)$. Moreover, the diaryl heterocycles template is considered as a typical model of selective inhibitor of COX-2 isozyme (6). Quinazoline derivatives with either halogen- or electron-rich substituent at 6th or 7th position are known to promote activity against bacteria and inflammation (7). Proquazone 1 (Fig. 1) is chemically known as 1-isopropyl-7-methyl-4-phenylquinazolin-2(1H)-one, which is chemically quinazoline derivative, has been known to have an excellent non-steroidal anti-inflammatory effect resulting from its anti-COX-2effect, therefore it was often used in the treatment of rheumatoid arthritis, osteoarthritis and other chronic inflammatory diseases (8). 


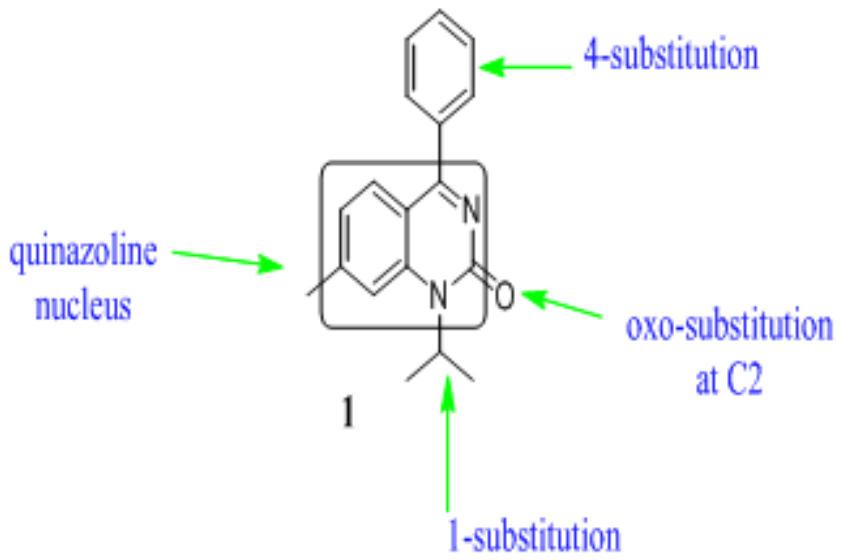

Figure 1: Structure activity relationship (SAR) of proquazone.

In the current study, as a complement to our previous work (9), we aimed to design and synthesize a novel series of 1-(4Acetylphenyl)-7,7-dimethyl-3-(substitutedphenyl)-1,2,3,4,7,8-octahydroquinazolin-5(6H)-ones (6-15) which devoid acidic properties with the accompanied common NSAIDs side effects and at the same time provide the required molecular bulkiness that prevent the compounds to enter the narrow COX-1 binding site. Furthermore, the design takes into account that the synthesized compounds will have molecular weights which will not affect their oral absorption. Molecular hybridization strategy (10) was used to design the target compounds 6-15 as active anti-inflammatoryagents with reduced adverse effects (Fig. 2).

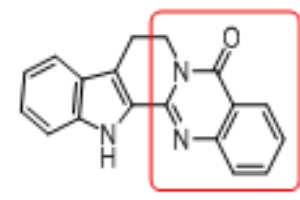

2

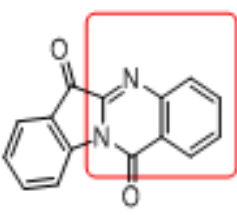

3

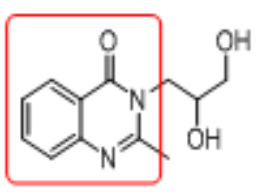

Diproqualone Synthetic anti-inflammatory

Natural anti-inflammatory

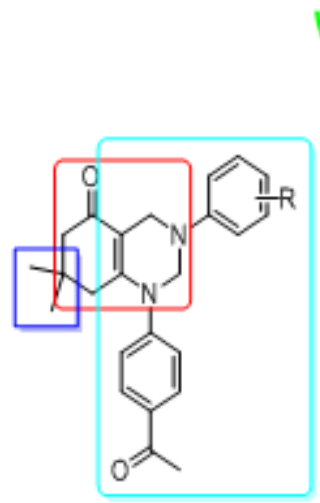

6-15

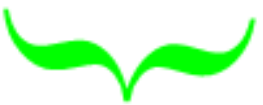

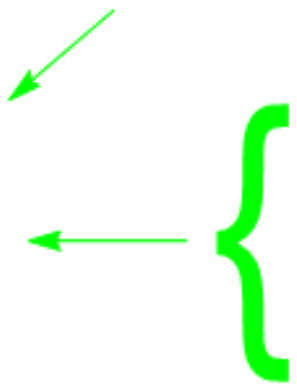

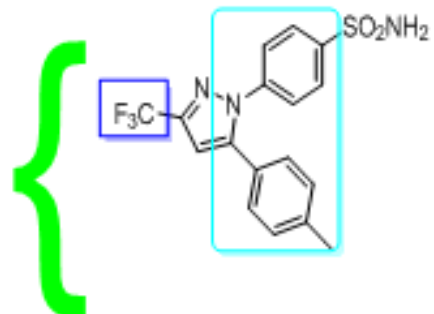

Celecoxib

Synthetic selective COX-2

Figure 2: Rational design of the target quinazolinone derivatives (6-15).

\section{RESULTS AND DISCUSSIONS}

\subsection{Chemistry}

In this work, as a complement to our previous work (9), the novel key intermediate enaminone $\mathbf{5}$ was prepared by condensation of equimolar amounts of 5,5-dimethyl-1,3-cyclohexanedione (dimedone) 4 with 4-aminoacetophenone via heating the reactants under reflux in toluene using our reported method (9). Toluene provides the reaction with a relatively higher temperature necessary for the completion of the reaction.

The novel octahydro-5-oxoquinazolines (6-15) were obtained in high yields (65-85\%) using one-pot reaction (threecomponent reaction) by refluxing a mixture of enaminone $\mathbf{5}$, a primary aromatic amine and formalin (two moles) in ethanol containing catalytic amount of acetic acid (Scheme 1) (9). 
<smiles>CC1(C)CC(=O)C=C(O)C1</smiles>

(4)

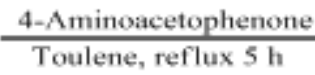<smiles>CC(=O)c1ccc(NC2=CC(=O)CC(C)(C)C2)cc1</smiles>

(5)

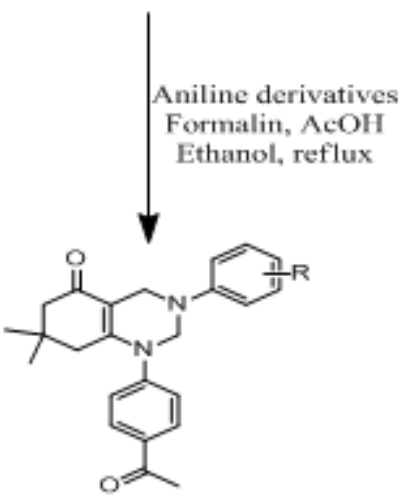

(6-15)

Scheme 1: The preparation of the key starting enaminone 5 to get the required quinazolinone (6-15) derivatives.

The novel octahydro-5-oxoquinazoline derivatives (6-15) were formed via a Mannich reaction on C-2 atom of the enaminone system (active methylene), i.e. nucleophilic addition followed by ring formation using the second molecule of formaldehyde.

The new compounds were confirmed using High Resolution Mass Spectrometry and elemental analyses and various spectroscopic methods. $1 \mathrm{H}-\mathrm{NMR}$ spectrum proved the disappearance of both singlets at $\delta=7.85 \mathrm{ppm}$ (NH group) and at $\delta=5.65$ (vinylic proton) of starting enaminone 5 and appearance of two characteristic singlets at $\delta=4.88$ and $4.21 \mathrm{ppm}$ indicating the formation of two methylene groups at 2- and 4-position of the quinazoline skeleton. ${ }^{13} \mathrm{C}-\mathrm{NMR}$ spectra proved the proposed 5-oxo-octahydroquinazolin structures due to the appearance of characteristic peaks around $\delta=69.68$ and 50.35 ppm which were assignable to C2- and C4- of the quinazoline nucleus.

\subsection{COX-1/2 inhibitory activities:}

The synthesized quinazolinones (6-15)were screened for their in vitro anti-inflammatory activity using Celecoxib (CEL), Diclofenac sodium (DCF) and Indomethacin (INM) as reference drugs. The ovine cyclooxygenase enzyme (COX) inhibition assay directly measures prostaglandin $\mathrm{F}_{2} \alpha\left(\mathrm{PGF}_{2} \alpha\right)$ by $\mathrm{SnCl}_{2}$ reduction of COX-derived prostaglandin $\mathrm{H}_{2}(\mathrm{PGH})$ produced in the COX reaction. The results listed in Table 1.

Table 1: In vitro COX-1, COX-2 inhibitory activities of derivatives 6-15 against celecoxib, diclofenac and indomethacin as reference drugs. Data are presented as mean \pm SD $(n=3)$.

\begin{tabular}{|c|c|c|c|}
\hline Compound & COX-1 IC50 $(\mu \mathrm{M})$ & COX-2 IC50 $(\mu \mathrm{M})$ & $\begin{array}{l}\text { COX-2 Selective } \\
\text { Index (S.I.)* }\end{array}$ \\
\hline Celecoxib(CEL) & $0.05 \pm 0.02$ & $14.8 \pm 4.08$ & 296 \\
\hline Diclofenac sodium(DCF) & $0.8 \pm 0.34$ & $3.9 \pm 0.88$ & 4.87 \\
\hline Indomethacin(IND) & $0.49 \pm 0.10$ & $0.039 \pm 0.01$ & 0.08 \\
\hline 6 & $0.22 \pm 0.08$ & $3.12 \pm 1.05$ & 14.18 \\
\hline 7 & $0.56 \pm 0.12$ & $6.54 \pm 2.43$ & 11.68 \\
\hline 8 & $1.23 \pm 0.35$ & $9.87 \pm 3.52$ & 8.02 \\
\hline 9 & $0.98 \pm 0.17$ & $7.41 \pm 2.86$ & 7.56 \\
\hline 10 & $1.16 \pm 0.28$ & $11.2 \pm 2.88$ & 9.66 \\
\hline 11 & $0.74 \pm 0.11$ & $5.23 \pm 1.78$ & 7.07 \\
\hline 12 & $0.63 \pm 0.09$ & $4.86 \pm 1.56$ & 7.71 \\
\hline 13 & $1.42 \pm 0.18$ & $9.56 \pm 3.25$ & 6.73 \\
\hline 14 & $1.39 \pm 0.19$ & $8.56 \pm 2.96$ & 6.16 \\
\hline 15 & $0.57 \pm 0.08$ & $4.62 \pm 1.12$ & 8.11 \\
\hline
\end{tabular}


The results showed that the reference drugs; celecoxib (CEL), diclofenac (DCF) and indomethacin (INM) exhibited COX-1 inhibitory activity with IC50 14.8, 3.9 and $0.039 \mu \mathrm{M}$ respectively and COX-2 inhibitory activity with IC50 $0.05,0.8$ and 0.49 $\mu \mathrm{M}$ respectively.

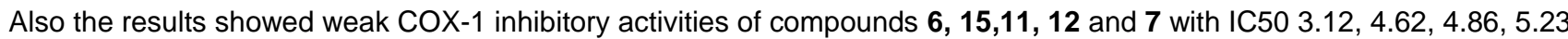
and $6.54 \mu \mathrm{M}$ respectively. On the contrary those compounds exhibited good COX-2 inhibitory activities with IC50 0.22 , $0.56,0.57,0.74$ and $0.63 \mu \mathrm{M}$ respectively. In addition, the results showed a significant activities of compounds $9,10,8,14$ and 13 with IC50 $0.98,0.1 .16,1.23,1.39$ and $1.42 \mu \mathrm{M}$ respectively.

Furthermore, the results showed that the highest COX-2 selectivity index S.I. of compounds $6, \mathbf{7}, \mathbf{1 0}, \mathbf{1 5}, \mathbf{8}, \mathbf{1 2}, \mathbf{9}, \mathbf{1 1}, \mathbf{1 3}$ and 14 equal to $14.18,11.68,9.66,8.11,8.02,7.71,7.56,7.08,6.73$ and 6.16 respectively which are higher than Diclofenac DCF (S.I. = 4.87) and more than 77 folds higher than Indomethacin IND (S.I. = 0.08). As shown in Table 1.

\subsection{Docking studies:}

Docking studies have been carried out to explore the ability of the most potent and most selective synthesized compound 6 to bind to the COX-1 and COX-2 receptors at the same active site as reported in the literature (11).

Redocking of the known cyclooxygenase-1 inhibitor Meloxicam in the COX-1 crystal structure (PDB: 4O1Z). As shown in (Fig. 3) And the known cyclooxygenase-2 inhibitor Celecoxib in the COX-2 crystal structure (PDB: 3LN1). As shown in (Fig. 4) Showed Root-mean-square deviation (RMSD) values of less than 2 (1.891) and (1.435), respectively; which indicates the confidence in the produced docking results.

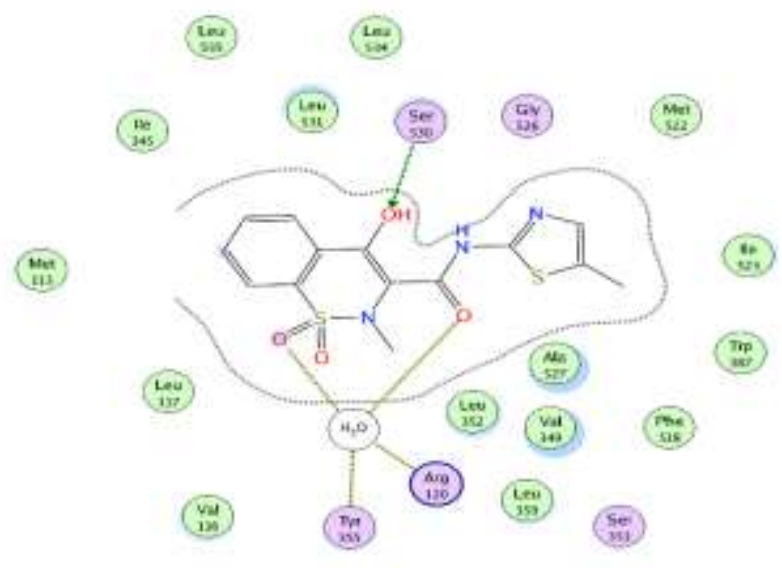

Figure 3. Meloxicam interaction with COX-1 isozyme.

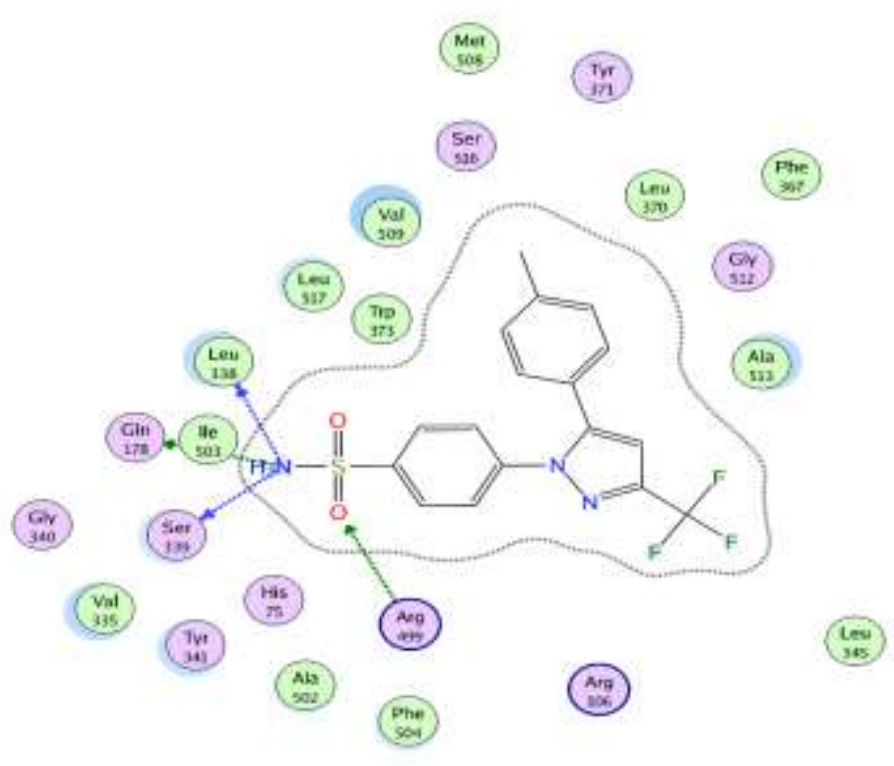

Figure 4. Celecoxib interaction with COX-2 isozyme.

The docking of compound 6 in COX-1 and COX-2 isozymes showed good fit in the active site of COX-2 (Fig. 5b) receptor rather than the active site of COX-1 (Fig. 5a) mainly due to the bulk size of the tested compound which hindered it from good fitting in smaller COX-1 active site. 
(a)

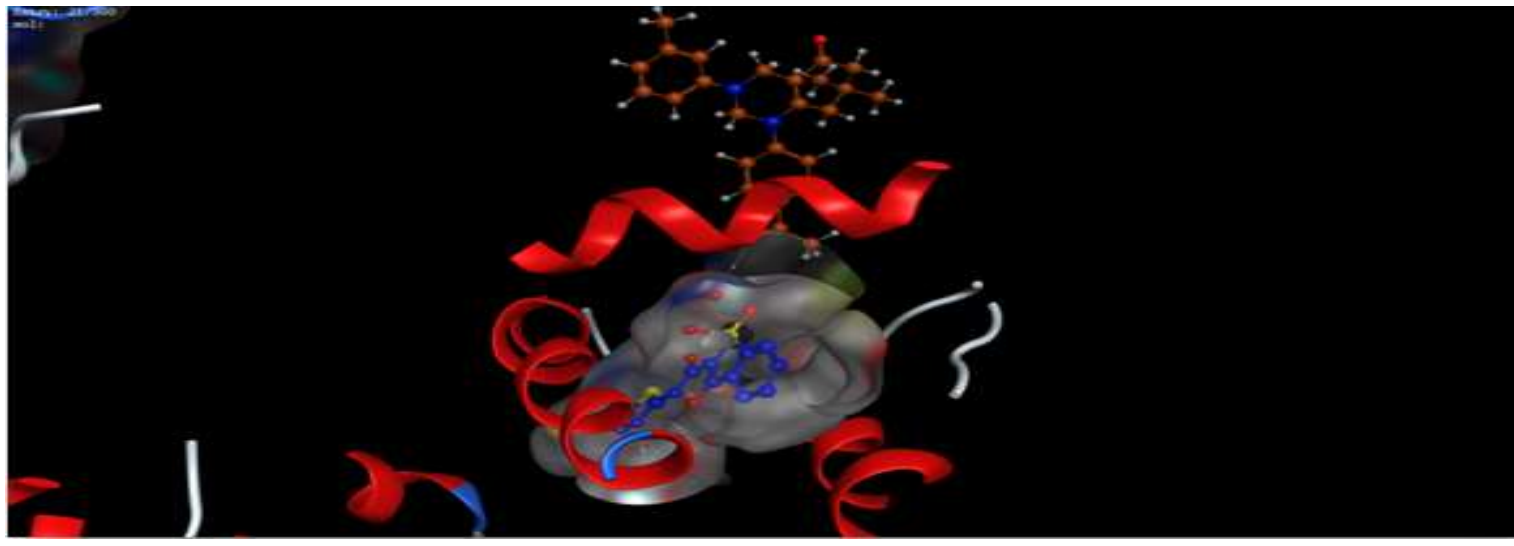

(b)

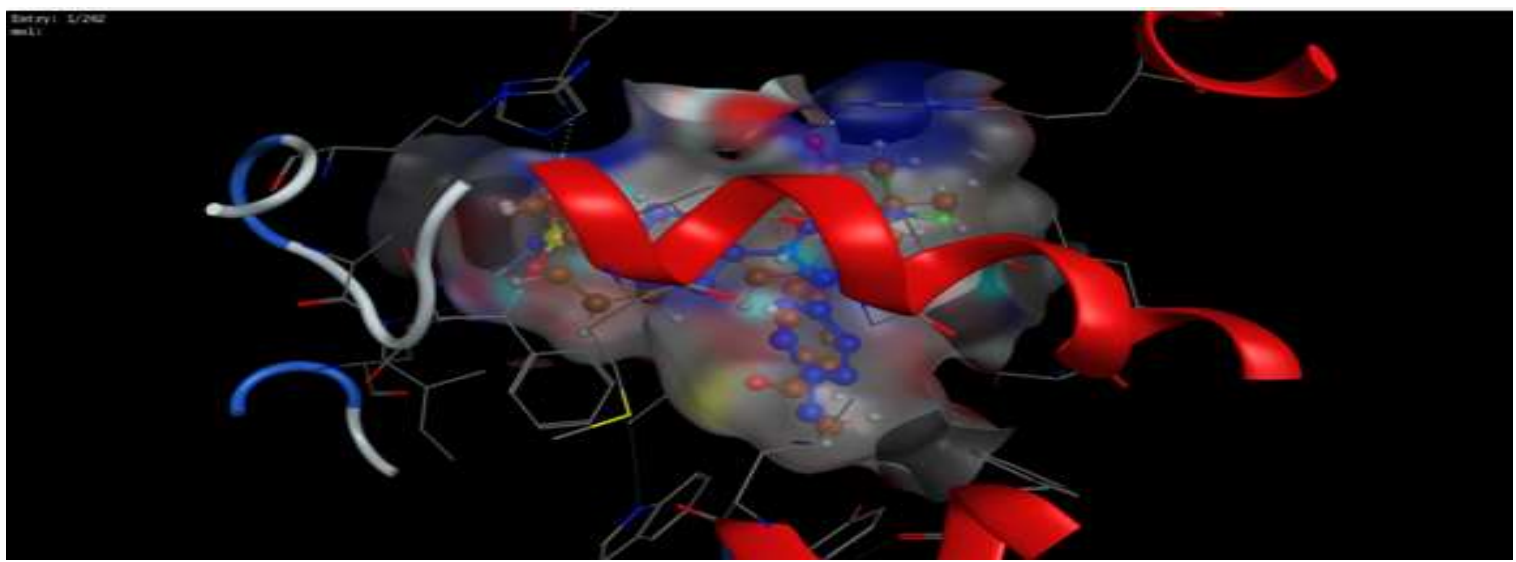

Fig. 5. (a) 3D structure of COX-1 enzyme showing fitting of meloxicam (blue ligand) in the active site and the lack of fitting of compound 6 (red ligand) (b) 3D structure of COX-2 enzyme showing fitting of celecoxib (blue ligand) in the active site and the fitting of compound $\mathbf{6}$ (red ligand)

The binding scores of compound 6 are -7.7779 and -5.6361 in COX-2 and COX-1 respectively. The interaction of compound 6 in COX-2 (Fig.6) active site showed interaction with key active site residues; Leu 338, Ser 339, Tyr 341, Arg 499, Phe 504 and Val 509. In addition, compound 6 showed interaction with residues Val 335 and Ser 516 . Compound 6 exhibits hydrophobic interaction with residues Val 335, Leu 338, Ser 339, Tyr 341, Phe 504 and Val 509. Also Compound 6 showed pi- $H$ interaction with Ser 339 with a distance of $3.70{ }^{\circ} \mathrm{A}$. Furthermore, compound 6 showed hydrogen bonding between Arg 499 and the oxygen atom of the acetyl group with a distance of $3.40{ }^{\circ} \mathrm{A}$ and Ser 516 with the oxygen atom of the carbonyl group on atom number 5 of the quinazoline ring with a distance of $2.77^{\circ} \mathrm{A}$.

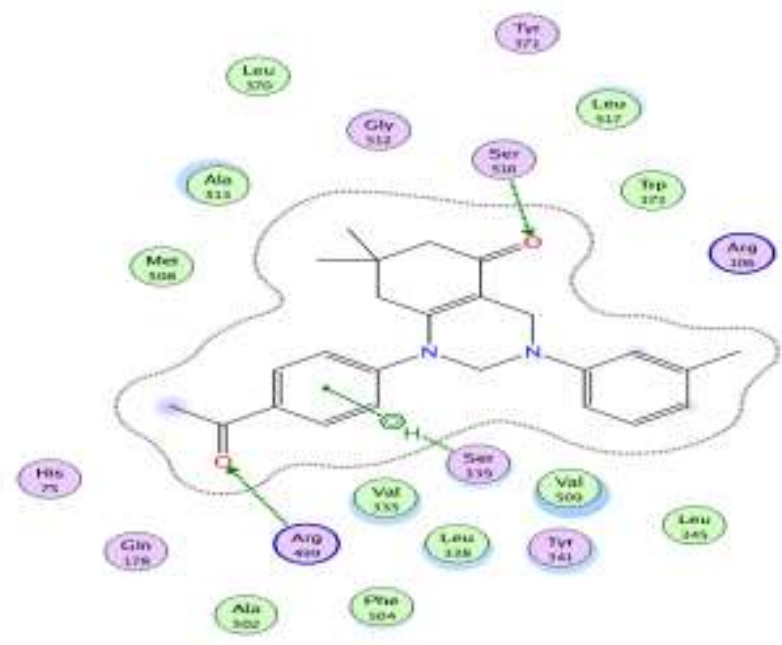

Figure 6. Ligand Interaction between compound 6 and COX-2 isozyme. 


\section{EXPERIMENTAL:}

\subsection{Chemistry:}

Melting points were determined with a Gallenkamp (London, U.K.) melting point apparatus and are uncorrected. Infrared (IR) spectra were recorded in potassium bromide $(\mathrm{KBr})$ discs using Bruker Vector, 22FT-IR Fourier Transform Infrared (FTIR) spectrometer (Germany), and expressed in wave number v ; $\left(\mathrm{cm}^{-1}\right)$. NMR Spectra (NMR) Spectra were taken using a Varian Unity INOVA $400 \mathrm{MHz}$ for proton and $101 \mathrm{MHz}$ for carbon; university of Aberdeen, UK. All numbers referring to NMR data obtained are in parts per million (ppm).

High Resolution Mass Spectra (HRESI-MS Spectra) were obtained using the Thermo Instruments MS system (LTQ XL/LTQ Orbitrap Discovery) coupled to a Thermo Instruments HPLC system (Accela PDA detector, Accela PDA autosampler and Pump) at university of Aberdeen, UK. Elemental analyses were determined using Vario EL III German CHN Elemental analyzer model, Regional Centre for Mycology and Biotechnology, Al-Azhar University, Cairo, Egypt. All the results of elemental analyses corresponded to the calculated values within experimental error.

Chemicals and solvents used in the preparation of the target compounds are of commercial grade and purchased from Sigma Aldrich, Alfa Aesar, Merck, Fluka. Chemicals and solvents were used without purification. Thin layer chromatography (TLC) was used for monitoring chemical reaction and was carried out using silica gel 60 F254 precoated sheet $2020 \mathrm{~cm}$, layer thickening $0.2 \mathrm{~mm}$ (E, Merck, Germany), and were visualized using UV - lamp at $254 \mathrm{~nm}$.

\subsubsection{3-(4-Acetyl-phenylamino)-5,5-dimethyl-cyclohex-2-enone (5)}

A mixture of equimolar amounts of 5, 5-dimethylcyclohexan-1,3-dione (dimedone) (1.40 $\mathrm{g}, 10 \mathrm{mmol}$ ) and $\mathrm{P}$ aminoacetophenone $(1.35 \mathrm{~g}, 10 \mathrm{mmol})$ in toluene $(50 \mathrm{~mL})$ was heated under reflux for $5 \mathrm{~h}$. The reaction mixture was concentrated and allowed to cool to room temperature. The obtained crystalline product (I) was filtered, dried and crystallized from ethanol. Yield: $7 \mathrm{~g}(87 \%)$, m.p. $210-212^{\circ} \mathrm{C}$ as reported (9).

\subsubsection{1-(4-Acetylphenyl)-7,7-dimethyl-3-(substitutedphenyl)-1,2,3,4,7,8- octahydroquinazolin-5(6H)-ones (6-15).}

To a solution of 3-(4-acetylphenyl amino)-5, 5-dimethylcyclohex-2-enone (5) (0.438 g, $1.70 \mathrm{mmol})$ in ethanol (30 $\mathrm{mL})$, an appropriate aromatic amine $(1.70 \mathrm{mmol})$, formaldehyde solution $40 \%(0.204 \mathrm{~mL}, 3.40 \mathrm{mmol})$ and glacial acetic acid $(10$ drops) were added. The reaction mixture was heated under reflux for the proper time (Table 2). The reaction mixture was diluted with water $(40 \mathrm{~mL})$, basified with $\mathrm{NH}_{4} \mathrm{OH}$ to $\mathrm{pH}$ 8, and then left in a refrigerator for $3 \mathrm{~h}$. The separated precipitates were filtered, washed with water and crystallized from ethyl acetate/n-hexane (3:2), the physical data of the separated products (6-15) are shown in Table 2.

\subsubsection{1. quinazolin-5-one (6). \\ 1-(4-Acetyl-phenyl)-7,7-dimethyl-3-m-tolyl-2,3,4,6,7,8-octahydro-1H-}

1H NMR (400 MHz, CDCl3): $\delta$ ppm 7.90-6.60 (m, 8H, ArH), 4.88 (s, 2H, 2-CH2), 4.21 (s, 2H, 4-CH2), 2.53 (s, 3H, $\mathrm{COCH} 3), 2.19(\mathrm{~s}, 2 \mathrm{H}, 8-\mathrm{CH} 2), 2.17(\mathrm{~s}, 3 \mathrm{H}, \mathrm{CH} 3), 2.04(\mathrm{~s}, 2 \mathrm{H}, 6-\mathrm{CH} 2), 0.91(\mathrm{~s}, 6 \mathrm{H}, 2 \mathrm{CH} 3) .13 \mathrm{C} \mathrm{NMR}(101 \mathrm{MHz}, \mathrm{CDCl} 3)$ : $\delta$ ppm $196.7(5-\mathrm{C}=\mathrm{O}), 194.7(\mathrm{COCH} 3)$ 155.9-114.6 ( $\mathrm{C}^{\mathrm{sp} 2}+$ phenyl-C), $107.8\left(\mathrm{C}^{\mathrm{sp} 2}\right), 69.7(2-\mathrm{CH} 2), 50.4(4-\mathrm{CH} 2), 46.1(6-$ $\mathrm{CH} 2), 41.4(8-\mathrm{CH} 2), 33.1(\mathrm{C}(\mathrm{CH} 3) 2), 28.3(2 \mathrm{CH} 3), 26.7\left(\mathrm{COCH}_{3}\right), 21.7\left(\mathrm{CH}_{3}\right)$. HRESI-MS m/z calculated for $[\mathrm{M}+\mathrm{Na}]^{+}$ $\mathrm{C}_{25} \mathrm{H}_{28} \mathrm{~N}_{2} \mathrm{NaO}_{2}: 411.2043$, found: 411.2029.

\subsubsection{1-(4-Acetyl-phenyl)-3-(4-chloro-phenyl)-7,7-dimethyl-2,3,4,6,7,8-octahydro-1H- quinazolin-5-one (7).}

${ }^{1} \mathrm{H}$ NMR (400 MHz, $\left.\mathrm{CDCl}_{3}\right): \delta \mathrm{ppm} 7.96(\mathrm{~d}, J=8.0 \mathrm{~Hz}, 2 \mathrm{H}, \mathrm{ArH}), 7.15(\mathrm{~d}, J=8.0 \mathrm{~Hz}, 2 \mathrm{H}, \operatorname{ArH}), 7.04(\mathrm{~d}, J=12.0 \mathrm{~Hz}, 2 \mathrm{H}$, $\mathrm{ArH}), 6.81(\mathrm{~d}, J=12.0 \mathrm{~Hz}, 2 \mathrm{H}, \mathrm{ArH}), 4.94\left(\mathrm{~s}, 2 \mathrm{H}, 2-\mathrm{CH}_{2}\right), 4.26\left(\mathrm{~s}, 2 \mathrm{H}, 4-\mathrm{CH}_{2}\right), 2.60\left(\mathrm{~s}, 3 \mathrm{H}, \mathrm{COCH}_{3}\right), 2.25(\mathrm{~s}, 2 \mathrm{H}, 8-$ $\left.\mathrm{CH}_{2}\right), 2.09\left(\mathrm{~s}, 2 \mathrm{H}, 6-\mathrm{CH}_{2}\right), 0.96\left(\mathrm{~s}, 6 \mathrm{H}, 2 \mathrm{CH}_{3}\right) .{ }^{13} \mathbf{C ~ N M R}\left(101 \mathrm{MHz}, \mathbf{C D C l}_{3}\right)$ : $\delta$ ppm $196.8(5-\mathrm{C}=\mathrm{O}), 194.8(\underline{\mathrm{COCH}}), 156.1-$ $118.9\left(\mathrm{C}^{\mathrm{sp2}}+\right.$ phenyl-C), $107.3\left(\mathrm{C}^{\mathrm{sp2}}\right), 69.8\left(2-\mathrm{CH}_{2}\right), 50.4\left(4-\mathrm{CH}_{2}\right), 46.1\left(6-\mathrm{CH}_{2}\right), 41.4\left(8-\mathrm{CH}_{2}\right), 33.2\left(\mathrm{C}\left(\mathrm{CH}_{3}\right)_{2}\right), 28.4\left(2 \mathrm{CH}_{3}\right)$, $26.7\left(\mathrm{COCH}_{3}\right)$. HRESI-MS m/z calculated for $[\mathrm{M}+\mathrm{Na}]^{+} \mathrm{C}_{24} \mathrm{H}_{25} \mathrm{CIN}_{2} \mathrm{NaO}_{2}: 431.1497$, found: 431.1480 .

\subsubsection{1-(4-Acetyl-phenyl)-3-(3-bromo-phenyl)-7,7-dimethyl-2,3,4,6,7,8-octahydro-1H- quinazolin-5-one (8).}

${ }^{1} \mathbf{H}$ NMR (400 MHz, $\left.\mathbf{C D C l}_{3}\right): \delta \mathrm{ppm} 7.96(\mathrm{~d}, J=12.0 \mathrm{~Hz}, 2 \mathrm{H}, \mathrm{ArH}), 7.26(\mathrm{~d}, J=8.0 \mathrm{~Hz}, 2 \mathrm{H}, \operatorname{ArH}), 7.04(\mathrm{~d}, J=8.0 \mathrm{~Hz}, 2 \mathrm{H}$, $\mathrm{ArH}), 6.74(\mathrm{~d}, J=8.0 \mathrm{~Hz}, 2 \mathrm{H}, \mathrm{ArH}), 4.93\left(\mathrm{~s}, 2 \mathrm{H}, 2-\mathrm{CH}_{2}\right), 4.26\left(\mathrm{~s}, 2 \mathrm{H}, 4-\mathrm{CH}_{2}\right), 2.60\left(\mathrm{~s}, 3 \mathrm{H}, \mathrm{COCH}_{3}\right), 2.24(\mathrm{~s}, 2 \mathrm{H}, 8-$ $\left.\mathrm{CH}_{2}\right), 2.09\left(\mathrm{~s}, 2 \mathrm{H}, 6-\mathrm{CH}_{2}\right), 0.96\left(\mathrm{~s}, 6 \mathrm{H}, 2 \mathrm{CH}_{3}\right) .{ }^{13} \mathbf{C}$ NMR (101 MHz, CDCl $\left.\mathbf{C O}_{3}\right): \delta$ ppm $196.8(5-\mathrm{C}=\mathrm{O}), 194.8\left(\underline{C O C H}_{3}\right), 156.1-$ $113.2\left(\mathrm{C}^{\mathrm{sp2}}+\right.$ phenyl-C), $107.3\left(\mathrm{C}^{\mathrm{sp} 2}\right), 69.6\left(2-\mathrm{CH}_{2}\right), 50.3\left(4-\mathrm{CH}_{2}\right), 46.0\left(6-\mathrm{CH}_{2}\right), 41.4\left(8-\mathrm{CH}_{2}\right), 33.1\left(\mathrm{C}\left(\mathrm{CH}_{3}\right)_{2}\right), 28.4\left(2 \mathrm{CH}_{3}\right)$, $26.7\left(\mathrm{COCH}_{3}\right)$. HRESI-MS m/z calculated for $[\mathrm{M}+\mathrm{Na}]^{+} \mathrm{C}_{24} \mathrm{H}_{25} \mathrm{BrN}_{2} \mathrm{NaO}_{2}: 475.0992$, found 475.0976 . 
3.1.2.4. 1-(4-Acetyl-phenyl)-3-(3-chloro-phenyl)-7,7-dimethyl-2,3,4,6,7,8-octahydro-1Hquinazolin-5-one (9).

${ }^{1} \mathrm{H}$ NMR (400 MHz, CDCl$)$ ) $\delta$ ppm7.94 (d, J=8.0 Hz, 2H, ArH), 7.09-6.69 (m, $\left.6 \mathrm{H}, \mathrm{ArH}\right), 4.90\left(\mathrm{~s}, 2 \mathrm{H}, 2-\mathrm{CH}_{2}\right), 4.22(\mathrm{~s}, 2 \mathrm{H}$, 4- $\left.\mathrm{CH}_{2}\right), 2.56\left(\mathrm{~s}, 3 \mathrm{H}, \mathrm{COCH}_{3}\right), 2.21\left(\mathrm{~s}, 2 \mathrm{H}, 8-\mathrm{CH}_{2}\right), 2.08\left(\mathrm{~s}, 2 \mathrm{H}, 6-\mathrm{CH}_{2}\right), 0.93\left(\mathrm{~s}, 6 \mathrm{H}, 2 \mathrm{CH}_{3}\right) .{ }^{13} \mathbf{C ~ N M R}(101 \mathbf{~ M H z}, \mathbf{C D C l} 3): \delta$ ppm $196.7(5-\mathrm{C}=\mathrm{O}), 194.6\left(\mathrm{COCH}_{3}\right), 156.0-115.3\left(\mathrm{C}^{\mathrm{sp} 2}+\right.$ phenyl-C), $107.4\left(\mathrm{C}^{\mathrm{sp} 2}\right), 68.9\left(2-\mathrm{CH}_{2}\right), 50.2\left(4-\mathrm{CH}_{2}\right), 46.0(6-$ $\left.\mathrm{CH}_{2}\right), 41.2\left(8-\mathrm{CH}_{2}\right), 33.0\left(\mathrm{C}\left(\mathrm{CH}_{3}\right)_{2}\right), 28.2\left(2 \mathrm{CH}_{3}\right), 26.6\left(\mathrm{COCH}_{3}\right)$. HRESI-MS m/z calculated for $\left[\mathrm{M}+\mathrm{Na}^{+} \mathrm{C}_{24} \mathrm{H}_{25} \mathrm{CIN}_{2} \mathrm{NaO}_{2}\right.$ 431.1497, found: 431.1480 .

\subsubsection{1-(4-Acetyl-phenyl)-3-(4-bromo-phenyl)-7,7-dimethyl-2,3,4,6,7,8-octahydro-1H- quinazolin-5-one (10).}

${ }^{1} \mathbf{H}$ NMR (400 MHz, $\mathbf{C D C l}_{3}$ ): $\delta \mathrm{ppm} 7.97(\mathrm{~d}, J=8.0 \mathrm{~Hz}, 2 \mathrm{H}, \mathrm{ArH}), 7.30(\mathrm{~d}, J=8.0 \mathrm{~Hz}, 2 \mathrm{H}, \mathrm{ArH}),, 7.05(\mathrm{~d}, \mathrm{~J}=8.0 \mathrm{~Hz}, 2 \mathrm{H}$, $\mathrm{ArH}), 6.76(\mathrm{~d}, J=8.0 \mathrm{~Hz}, 2 \mathrm{H}, \mathrm{ArH}), 4.95\left(\mathrm{~s}, 2 \mathrm{H}, 2-\mathrm{CH}_{2}\right), 4.27\left(\mathrm{~s}, 2 \mathrm{H}, 4-\mathrm{CH}_{2}\right), 2.61\left(\mathrm{~s}, 3 \mathrm{H}, \mathrm{COCH}_{3}\right), 2.26\left(\mathrm{~s}, 2 \mathrm{H}, 8-\mathrm{CH}_{2}\right)$,

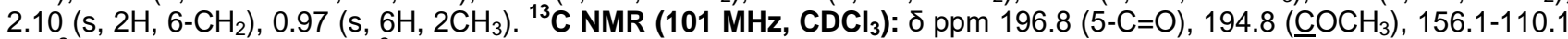

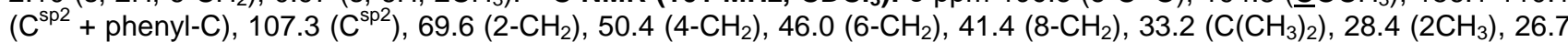
$\left(\mathrm{CO} \underline{\mathrm{CH}} \mathrm{H}_{3}\right)$. HRESI-MS $\mathrm{m} / \mathrm{z}$ calculated for $[\mathrm{M}+\mathrm{Na}]^{+} \mathrm{C}_{24} \mathrm{H}_{25} \mathrm{BrN}_{2} \mathrm{NaO}_{2}: 475.0992$, found 475.0976 .

\subsubsection{1-(4-Acetyl-phenyl)-3-(4-hydroxy-phenyl)-7,7-dimethyl-2,3,4,6,7,8-octahydro- 1H-quinazolin-5-one (11).}

${ }^{1} \mathrm{H}$ NMR (400 MHz, $\mathbf{C D C l}_{3}$ ): $\delta$ ppm $7.86(\mathrm{~d}, J=8.0 \mathrm{~Hz}, 2 \mathrm{H}, \mathrm{ArH}), 6.92(\mathrm{~d}, J=8.0 \mathrm{~Hz}, 2 \mathrm{H}, \mathrm{ArH}), 6.75-6.67(\mathrm{~m}, 5 \mathrm{H}, \mathrm{ArH}$ and $\mathrm{OH}), 4.84\left(\mathrm{~s}, 2 \mathrm{H}, 2-\mathrm{CH}_{2}\right), 4.18\left(\mathrm{~s}, 2 \mathrm{H}, 4-\mathrm{CH}_{2}\right), 2.52\left(\mathrm{~s}, 3 \mathrm{H}, \mathrm{COCH}_{3}\right), 2.20\left(\mathrm{~s}, 2 \mathrm{H}, 8-\mathrm{CH}_{2}\right), 1.98\left(\mathrm{~s}, 2 \mathrm{H}, 6-\mathrm{CH}_{2}\right), 0.85(\mathrm{~s}, 6 \mathrm{H}$, $\left.2 \mathrm{CH}_{3}\right) .{ }^{13} \mathrm{CNMR}\left(101 \mathrm{MHz}, \mathrm{CDCl}_{3}\right): \delta \mathrm{ppm} 197.1(5-\mathrm{C}=\mathrm{O}), 195.4\left(\mathrm{COCH}_{3}\right), 157.9-116.2\left(\mathrm{C}^{\mathrm{sp} 2}+\right.$ phenyl-C), $105.9\left(\mathrm{C}^{\mathrm{sp} 2}\right)$, $71.9\left(2-\mathrm{CH}_{2}\right), 49.9\left(4-\mathrm{CH}_{2}\right), 46.0\left(6-\mathrm{CH}_{2}\right), 41.4\left(8-\mathrm{CH}_{2}\right), 33.1\left(\mathrm{C}_{(\mathrm{C}} \mathrm{CH}_{3}\right), 28.3\left(2 \mathrm{CH}_{3}\right), 26.7\left(\mathrm{COCH}_{3}\right)$. HRESI-MS m/z calculated for $[\mathrm{M}+\mathrm{Na}]^{+} \mathrm{C}_{24} \mathrm{H}_{26} \mathrm{~N}_{2} \mathrm{NaO}_{3}: 413.1836$, found 413.1830 .

\subsubsection{1-(4-Acetyl-phenyl)-3-(4-fluoro-phenyl)-7,7-dimethyl-2,3,4,6,7,8-octahydro-1H- quinazolin-5-one (12).}

${ }^{1} \mathrm{H}$ NMR $\left(400 \mathrm{MHz}, \mathbf{C D C l}_{3}\right)$ : $\delta$ ppm $7.95(\mathrm{~d}, J=8.0 \mathrm{~Hz}, 2 \mathrm{H}, \mathrm{ArH}), 7.02-6.94(\mathrm{~m}, 2 \mathrm{H}, \mathrm{ArH}), 6.92-6.84(\mathrm{~m}, 4 \mathrm{H}, \mathrm{ArH}), 4.92$ $\left(\mathrm{s}, 2 \mathrm{H}, 2-\mathrm{CH}_{2}\right), 4.26\left(\mathrm{~s}, 2 \mathrm{H}, 4-\mathrm{CH}_{2}\right), 2.60\left(\mathrm{~s}, 3 \mathrm{H}, \mathrm{COCH}_{3}\right), 2.27\left(\mathrm{~s}, 2 \mathrm{H}, 8-\mathrm{CH}_{2}\right), 2.10\left(\mathrm{~s}, 2 \mathrm{H}, 6-\mathrm{CH}_{2}\right), 0.98\left(\mathrm{~s}, 6 \mathrm{H}, 2 \mathrm{CH}_{3}\right) .{ }^{13} \mathrm{C}$ NMR (101 MHz, CDCl 3$)$ : $\delta$ ppm $196.8(5-\mathrm{C}=\mathrm{O}), 194.8\left(\mathrm{COCH}_{3}\right), 156.1-115.7\left(\mathrm{C}^{\mathrm{sp} 2}+\right.$ phenyl-C) $), 107.2\left(\mathrm{C}^{\mathrm{sp} 2}\right), 70.9\left(2-\mathrm{CH}_{2}\right)$, $50.4\left(4-\mathrm{CH}_{2}\right), 46.4\left(6-\mathrm{CH}_{2}\right), 41.5\left(8-\mathrm{CH}_{2}\right), 33.2\left(\mathrm{C}\left(\mathrm{CH}_{3}\right)_{2}\right), 28.4\left(2 \mathrm{CH}_{3}\right), 26.7\left(\mathrm{COCH}_{3}\right)$. HRESI-MS m/z calculated for $[\mathrm{M}+\mathrm{Na}]^{+} \mathrm{C}_{24} \mathrm{H}_{25} \mathrm{FN}_{2} \mathrm{NaO}_{2}: 415.1792$, found: 415.1797 .

\subsubsection{1-(4-Acetyl-phenyl)-3-(4-methoxy-phenyl)-7,7-dimethyl-2,3,4,6,7,8-octahydro-} 1H-quinazolin-5-one (13).

${ }^{1} \mathbf{H}$ NMR (400 MHz, $\left.\mathbf{C D C l}_{3}\right): \delta$ ppm 7.89-6.90 (m, 4H, ArH), $6.89-6.76(\mathrm{~m}, 4 \mathrm{H}, \mathrm{ArH}), 4.94\left(\mathrm{~s}, 2 \mathrm{H}, 2-\mathrm{CH}_{2}\right), 4.25(\mathrm{~s}, 2 \mathrm{H}, 4-$ $\left.\mathrm{CH}_{2}\right), 3.71\left(\mathrm{~s}, 3 \mathrm{H}, \mathrm{OCH}_{3}\right), 2.58\left(\mathrm{~s}, 3 \mathrm{H}, \mathrm{COCH}_{3}\right), 2.30\left(\mathrm{~s}, 2 \mathrm{H}, 8-\mathrm{CH}_{2}\right), 2.14\left(\mathrm{~s}, 2 \mathrm{H}, 6-\mathrm{CH}_{2}\right), 1.02\left(\mathrm{~s}, 6 \mathrm{H}, 2 \mathrm{CH}_{3}\right) .{ }^{13} \mathrm{CNMR}(101$ $\left.\mathbf{M H z} \mathbf{C D C l}_{3}\right): \delta$ ppm $196.9(5-\mathrm{C}=\mathrm{O}), 194.7\left(\mathrm{COCH}_{3}\right), 156.1-111.5\left(\mathrm{C}^{\mathrm{sp} 2}+\right.$ phenyl-C), $108.13\left(\mathrm{C}^{\mathrm{sp2}}\right), 69.9\left(2-\mathrm{CH}_{2}\right), 55.6$

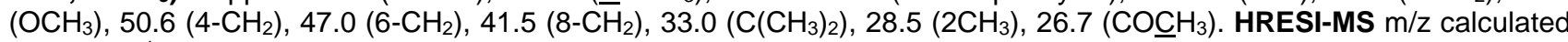
for $[\mathrm{M}+\mathrm{Na}]^{+} \mathrm{C}_{25} \mathrm{H}_{28} \mathrm{~N}_{2} \mathrm{NaO}_{3}: 427.1992$, found 427.1979.

\subsubsection{1-(4-Acetyl-phenyl)-7,7-dimethyl-3-p-tolyl-2,3,4,6,7,8-octahydro-1H-quinazolin- 5-one (14).}

${ }^{1} \mathrm{H}$ NMR (400 MHz, $\left.\mathrm{CDCl}_{3}\right)$ : $\delta$ ppm 7.98-7.95 (m, 3H, ArH), 7.09-7.04 (m, 2H, ArH), 6.72-6.67 (m, 3H, ArH), $4.96(\mathrm{~s}, 2 \mathrm{H}, 2-$ $\left.\mathrm{CH}_{2}\right), 4.29\left(\mathrm{~s}, 2 \mathrm{H}, 4-\mathrm{CH}_{2}\right), 2.61\left(\mathrm{~s}, 3 \mathrm{H}, \mathrm{COCH}_{3}\right), 2.27\left(\mathrm{~s}, 3 \mathrm{H}, \mathrm{CH}_{3}\right), 2.25\left(\mathrm{~s}, 2 \mathrm{H}, 8-\mathrm{CH}_{2}\right), 2.12\left(\mathrm{~s}, 2 \mathrm{H}, 6-\mathrm{CH}_{2}\right), 0.98(\mathrm{~s}, 6 \mathrm{H}$, $\left.2 \mathrm{CH}_{3}\right) .{ }^{13} \mathrm{C}$ NMR (101 MHz, $\left.\mathrm{CDCl}_{3}\right)$ : $\delta$ ppm $196.9(5-\mathrm{C}=\mathrm{O}), 194.8\left(\mathrm{COCH}_{3}\right)$ 156.0-114.6 $\left(\mathrm{C}^{\mathrm{sp2}}+\right.$ phenyl-C), $108.0\left(\mathrm{C}^{\mathrm{sp} 2}\right)$,

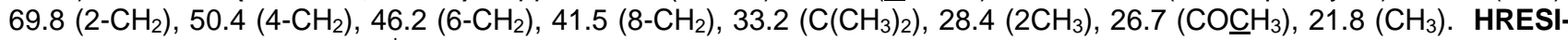
MS m/z calculated for $[\mathrm{M}+\mathrm{Na}]^{+} \mathrm{C}_{25} \mathrm{H}_{28} \mathrm{~N}_{2} \mathrm{NaO}_{2}: 411.2043$, found 411.2031.

3.1.2.10.

1-(4-Acetyl-phenyl)-7,7-dimethyl-3-phenyl-2,3,4,6,7,8-octahydro-1Hquinazolin-5-one (15).

${ }^{1} \mathrm{H}$ NMR $\left(400 \mathrm{MHz}, \mathrm{CDCl}_{3}\right): \delta \mathrm{ppm} 7.93(\mathrm{~d}, \mathrm{~J}=8.0 \mathrm{~Hz}, 2 \mathrm{H}, \mathrm{ArH}), 7.21-7.16(\mathrm{~m}, 2 \mathrm{H}, \mathrm{ArH}), 7.02(\mathrm{~d}, \mathrm{~J}=8.0 \mathrm{~Hz}, 2 \mathrm{H}, \mathrm{ArH})$, 6.89-6.83 (m, 3H, ArH), $4.95\left(\mathrm{~s}, 2 \mathrm{H}, 2-\mathrm{CH}_{2}\right), 4.28\left(\mathrm{~s}, 2 \mathrm{H}, 4-\mathrm{CH}_{2}\right), 2.57\left(\mathrm{~s}, 3 \mathrm{H}, \mathrm{COCH}_{3}\right), 2.23\left(\mathrm{~s}, 2 \mathrm{H}, 8-\mathrm{CH}_{2}\right), 2.09(\mathrm{~s}, 2 \mathrm{H}, 6-$ $\left.\mathrm{CH}_{2}\right), 0.95\left(\mathrm{~s}, 6 \mathrm{H}, 2 \mathrm{CH}_{3}\right) .{ }^{13} \mathrm{C}$ NMR (101 MHz, CDCl 3$): \delta \mathrm{ppm} 196.6(5-\mathrm{C}=\mathrm{O}), 194.5\left(\mathrm{COCH}_{3}\right), 155.9-117.4\left(\mathrm{C}^{\mathrm{sp} 2}+\right.$ phenyl-

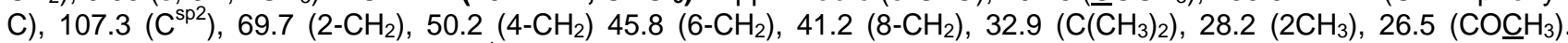
HRESI-MS $\mathrm{m} / \mathrm{z}$ calculated for $[\mathrm{M}+\mathrm{Na}]^{+} \mathrm{C}_{24} \mathrm{H}_{26} \mathrm{~N}_{2} \mathrm{NaO}_{2}: 397.1886$, found: 397.1876 .

\subsection{COX-1/2 inhibitory assay:}

\subsubsection{Methodology:}


The synthesized quinazolinones (6-15)were screened for their in vitro anti-inflammatory activity using Celecoxib (CEL), Diclofenac sodium (DCF) and Indomethacin (INM) as reference drugs. The ovine cyclooxygenase enzyme (COX) inhibition assay directly measures prostaglandin $\mathrm{F}_{2} \alpha\left(\mathrm{PGF}_{2} \alpha\right)$ by $\mathrm{SnCl}_{2}$ reduction of COX-derived prostaglandin $\mathrm{H}_{2}\left(\mathrm{PGH} \mathrm{H}_{2}\right)$ produced in the COX reaction. The prostanoid product is quantified via enzyme immunoassay (EIA) kit (catalog no. 560131, Cayman Chemical, Ann Arbor, MI, USA) according to the previously reported method (12). This assay is an excellent tool which can be used for general inhibitor screening, or to eliminate false positive leads generated by less specific methods (13).

The in vitro cyclooxygenase enzyme type one (COX-1) and cyclooxygenase enzyme type two (COX-2) isozymes inhibition measure the ability of tested compounds to inhibit ovine COX-1 and human recombinant COX-2 using an enzyme immunoassay (EIA) (13)

\subsection{Docking study:}

The docking studies were carried out on Dell Precision ${ }^{\mathrm{TM}}$ T3600 Workstation [Intel Xeon E5-1660 3.3GHz, 16GB 1600MHz DDR3, ECC RDIMM 1TB (7200RPM), 1GB NVIDIA Quadro 2000, Windows 7 Professional (64 Bit)].

Molecular Operating Environment (MOE) package version 2016.08 (Chemical Computing Group, Inc. Molecular Operating Environment (MOE). CCG, Montreal, Canada. http://www.chemcomp.com) was used for performing docking studies.

\subsubsection{Receptor Preparation:}

Docking procedures were performed using Crystal Structure of ovine Cyclooxygenase-1 and murine Cyclooxygenase-2 downloading their structures from the Protein Data Bank (PDB): PDB code $401 \mathrm{Z}$ at $2.4{ }^{\circ} \mathrm{A}$ resolution and $3 \mathrm{LN} 1$ at $2.4{ }^{\circ} \mathrm{A}$ resolution, respectively.

Protein structures were first repaired, Ramchandran plot was plotted to ascertain the health of protein and then appropriately protonated in the presence of ligands using the Protonate 3-D process in MOE (14).

The original 4O1Z PDB file contained crystallized Heme and N-Acetyl-D-Glucosamine beside the receptor chains and the bound Meloxicam. The receptor (first chain) and the bound Meloxicam were kept.

The original 3LN1 PDB file contained crystallized Heme, $\beta$-Octylglucoside and $N$-Acetyl- $D$-Glucosamine beside the receptor chains and the bound Celecoxib. The receptor (first chain) and the bound Celecoxib were kept.

\subsubsection{Ligand Preparation:}

Compound 6 was built in ChemBioDraw Ultra 12.0 for further preparation in the MOE. In MOE, the ligand prepared for docking through the following steps: hydrogens were added, conformational search has been run for compound 6 and the best conformers subjected to energy minimization was performed using the MMFF94 force field (15).

Steepest descent algorithm was used for minimization, followed by conjugate gradient method, until it reached an RMS (root mean square) gradient of $0.00001 \mathrm{kcal} / \mathrm{mol} /{ }^{\circ} \mathrm{A}$. A database of the ligand was generated for further docking studies in the target receptors.

\subsubsection{Docking Procedure:}

The standard protocol of the procedure in MOE 2016.08 was applied in this work. The Alpha Triangle placement which derives poses by random superposition of ligand atom triplets alpha sphere dummies in the receptor site is to determine the poses. The London dG scoring function estimates the free energy of binding of the ligand from a given pose.

The output database dock file was created with different poses for the ligand and arranged according to the final score function $(S)$, which is the score of the last stage that was not set to none. The database browser was used for the visual inspection of different poses for the ligand and the best poses were chosen.

\section{CONCLUSION:}

It can be concluded that new quinazolinone (6-15) derivatives were prepared starting from cyclic $\beta$-diketone namely, dimedone through one-pot reaction of the enaminone 5 with anilines and 2 moles of formalin. The anti-inflammatory activity of the novel compounds (6-15) was measured in vitro COX-1/2 inhibitory activities in comparison with celecoxib (CEL), diclofenac sodium (DCF) and indomethacin (INM) as reference drugs. It was found that quinazolinone 6 and 8 bearing electron-donating substituents at phenyl ring are the most potent ones in this series against COX-2 isozyme in a way that is superior to that of diclofenac sodium (DCF) and indomethacin (INM). Furthermore, the results showed that

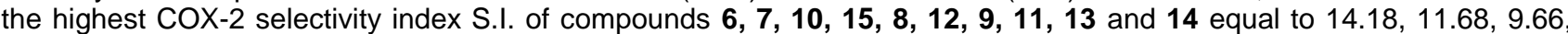
$8.11,8.02,7.71,7.56,7.08,6.73$ and 6.16 respectively which are higher than Diclofenac DCF $(S . I .=4.87)$ and more than 77 folds higher than Indomethacin IND (S.I. = 0.08).

\section{ACKNOWLEDGMENT:}

We gratefully acknowledge the support from Al-azhar University (Assuit branch), faculty of pharmacy, pharmaceutical organic department. 


\section{CONFLICT OF INTEREST:}

The authors declare that they have no conflict of interest.

This article does not contain any studies with human or animal participants performed by any of the authors.

\section{REFERENCES:}

1. A. A. Abdel-Aziz et al., Synthesis, anti-inflammatory, analgesic and COX-1/2 inhibition activities of anilides based on 5,5-diphenylimidazolidine-2,4-dione scaffold: Molecular docking studies. European journal of medicinal chemistry115, 121 (Jun 10, 2016).

2. A. Di Capua et al., Synthesis and biological evaluation of fluorinated 1,5-diarylpyrrole-3-alkoxyethyl ether derivatives as selective COX-2 inhibitors endowed with anti-inflammatory activity. European journal of medicinal chemistry109, 99 (Feb 15, 2016).

3. D. A. Patil, S. J. Surana, Synthesis, biological evaluation of 2,3-disubstituted-imidazolyl/benzimidazolylquinazolin-4(3H)-one derivatives. Medicinal Chemistry Research25, 1125 (2016).

4. M. S. Mosaad et al., Novel 6,8-dibromo-4(3H)-quinazolinone derivatives of promising anti-inflammatory and analgesic properties. Acta poloniae pharmaceutica67, 159 (Mar-Apr, 2010).

5. A. P. Nikalje, A. Ansari, S. Bari, V. Ugale, Synthesis, Biological Activity, and Docking Study of Novel Isatin Coupled Thiazolidin-4-one Derivatives as Anticonvulsants. Archiv der Pharmazie348, 433 (Jun, 2015).

6. A. Palomer et al., Identification of Novel Cyclooxygenase-2 Selective Inhibitors Using Pharmacophore Models. Journal of Medicinal Chemistry45, 1402 (2002/03/01, 2002).

7. A. K. Tiwari et al., Synthesis and pharmacological study of novel pyrido-quinazolone analogues as anti-fungal, antibacterial, and anticancer agents. Bioorganic \& Medicinal Chemistry Letters16, 4581 (9/1/, 2006).

8. A. A. Farghaly, A. A. Bekhit, J. Y. Park, Design and synthesis of some oxadiazolyl, thiadiazolyl, thiazolidinyl, and thiazolyl derivatives of $1 \mathrm{H}$-pyrazole as anti-inflammatory antimicrobial agents. Archiv der Pharmazie333, 53 (Mar, 2000).

9. O. I. el-Sabbagh, M. A. Shabaan, H. H. Kadry, E. S. Al-Din, New octahydroquinazoline derivatives: synthesis and hypotensive activity. European journal of medicinal chemistry45, 5390 (Nov, 2010).

10. C. Viegas-Junior, A. Danuello, V. da Silva Bolzani, E. J. Barreiro, C. A. Fraga, Molecular hybridization: a useful tool in the design of new drug prototypes. Current medicinal chemistry14, 1829 (2007).

11. R. G. Kurumbail et al., Structural basis for selective inhibition of cyclooxygenase-2 by anti-inflammatory agents. Nature384, 644 (Dec 19-26, 1996).

12. B. Roschek, Jr. et al., Pro-inflammatory enzymes, cyclooxygenase 1, cyclooxygenase 2, and 5-lipooxygenase, inhibited by stabilized rice bran extracts. Journal of medicinal food12, 615 (Jun, 2009).

13. M. A. Chowdhury et al., Synthesis of celecoxib analogues possessing a N-difluoromethyl-1,2-dihydropyrid-2-one 5-lipoxygenase pharmacophore: biological evaluation as dual inhibitors of cyclooxygenases and 5-lipoxygenase with anti-inflammatory activity. J Med Chem52, 1525 (Mar 26, 2009).

14. H. Singh, S. S. Marla, M. Agarwal, Docking studies of Tau protein. IAENG International Journal of Computer Science33, 36 (2007).

15. D. M. Elking, L. Fusti-Molnar, A. Nichols, Crystal structure prediction of rigid molecules. Acta Crystallographica Section B72, 488 (2016).

16. Marvin was used for drawing, displaying and characterizing chemical structures, substructures and reactions, Marvin 17.2.27, 2017, ChemAxon (http://www.chemaxon.com) 